\title{
The relationship between fasting plasma concentrations of selected flavonoids and their ordinary dietary intake
}

\author{
Jie Cao, Ying Zhang, Wei Chen and Xiujuan Zhao* \\ Department of Nutrition and Food Hygiene, Public Health School, Harbin Medical University, 194 Xuefu Road, Harbin, \\ Heilongjiang, People's Republic of China
}

(Received 24 April 2009 - Revised 21 July 2009 - Accepted 22 July 2009 - First published online 14 September 2009)

Epidemiological studies suggest that a diet high in flavonoids protects against chronic diseases such as CVD and cancer. The objective of the present study was to evaluate the relationship between the intake of quercetin, kaempferol, isorhamnetin, apigenin and luteolin and their corresponding plasma concentrations, and further to explore whether these flavonoids can serve as biomarkers of their intake. Flavonoid intake and their plasma concentrations were analysed in ninety-two subjects consuming their habitual diet. Flavonoid intake was estimated with 7-d dietary records using available data on the flavonoid content of food. Plasma flavonoid concentrations were quantified by HPLC. In addition, we undertook a dietary intervention study to investigate plasma apigenin concentration after the consumption of celery leaf. The mean intake estimates of quercetin, kaempferol, isorhamnetin, apigenin and luteolin amounted to 13.58, 14.97, 12.31, 4.23 and 8.08 mg/d, respectively. The corresponding mean plasma concentrations were $80.23,57 \cdot 86,39.94,10.62$ and $99.90 \mathrm{nmol} / \mathrm{l}$. The mean $7 \mathrm{~d}$ intake of five flavonoids was positively correlated to their corresponding plasma concentrations, with correlation coefficients ranging from $0 \cdot 33$ to $0 \cdot 51(P<0 \cdot 05)$. In the dietary intervention study, the plasma apigenin concentration rose after celery leaf ingestion, and fell within $28 \mathrm{~h}$ to below the limit of detection $(2.32 \mathrm{nmol} / \mathrm{l})$. The present results suggest that quercetin, kaempferol, isorhamnetin, apigenin and luteolin are bioavailable from the diet. The levels of fasting plasma flavonoids seem to be suitable biomarkers of short-term intake. The combination of plasma flavonoids with their intake may prove useful when the possible health-protective effects of flavonoids are studied.

Flavonols: Flavones: Diet: Plasma concentrations

Flavonoids are polyphenolic compounds with a $\mathrm{C}_{6}-\mathrm{C}_{3}-\mathrm{C}_{6}$ backbone and are an integral part of the human diet, these compounds are widespread in plant-derived foods such as vegetables, fruits, seeds and tea. Flavonoids are generally present in food as the $O$-glycosides and can be divided into flavonols, flavones, flavanones, catechins (flavanols), anthocyanidins, isoflavones, dihydroflavonols and chalcones according to their different chemical structures ${ }^{(1)}$. Within the subgroups of flavonols and flavones, the flavonol quercetin is the most frequently occurring compound in foods. Also common are kaempferol, isorhamnetin and the flavones apigenin and luteolin.

Recently, increasing attention has been paid to flavonoids because of their association with a wide range of different biological activities such as anti-bacterial, anti-inflammatory, anti-thrombotic, vasodilatory and anti-carcinogenic ${ }^{(2)}$. However, their investigation in epidemiological studies is hampered by difficulties in exposure assessment. A number of epidemiological studies have been carried out on the health effects of some flavonoids (flavonols, flavones, flavanols and isoflavones) and lignans ${ }^{(3)}$, which largely relied on the accurate estimation of polyphenol intake or exposure. So far, the restricted amount of epidemiological evidence on the roles of flavonoids in human disease prevention has produced conflicting results. On the correlation of flavonoids (mainly flavonols) with CVD risk, some prospective studies in human subjects have shown an inverse association ${ }^{(4,5)}$, whereas others have shown no association ${ }^{(6,7)}$. Studies related to cancer have also produced contradictory results ${ }^{(8,9)}$. Difficulties in assessing the exact exposure level are among the most important reasons for this inconsistency.

Accurate assessment of the relationship between the ingestion of flavonoid compounds and human health requires a food composition database that provides quantitative information on specific compounds in specific foods to support clinical and epidemiological studies ${ }^{(10)}$. However, limited availability of food composition data and bias inherent in dietary assessment methods made the estimation of dietary flavonoid intake difficulty, which can confound the ability to infer epidemiological relationships regarding health and disease. To obtain further insight into the health effects of flavonoids, reliable biomarkers for the flavonoid intake are needed. The most common approaches to studying biomarkers are the measurement of the original compound or its metabolites in blood and urine ${ }^{(11)}$. In fact, these methods have been successfully tested in studies in which the human diet has been supplemented with high-flavonoid foods ${ }^{(12,13)}$ or isolated compounds ${ }^{(14)}$. However, few data are available concerning

Abbreviation: 7-d DR, 7-d dietary records.

* Corresponding author: Xiujuan Zhao, fax +86 451 87502885, email xiujuan_zhao@sina.com 
plasma flavonoid concentrations in subjects following their habitual diets. In the search for biomarkers applicable to epidemiological studies, a combination of a single biomarker measurement together with long-term dietary intake estimates is urgently needed.

Therefore, in the present study, we chose five flavonoids, quercetin, isorhamnetin, kaempferol, apigenin and luteolin, the most important representatives of the flavonols and flavones. The average dietary intake of the five flavonoids was estimated by means of 7-d dietary records in combination with a database providing the flavonoid content of food, which had been previously established by the method of Cao et al. ${ }^{(15)}$. Flavonoids in plasma were quantified by means of HPLC. We compared the flavonoid intake with the corresponding fasting plasma concentration. Furthermore, we determined plasma apigenin concentration after celery leaf consumption in the human dietary intervention study.

\section{Materials and methods}

\section{Materials}

All chemicals were analytical grade or chromatographic grade. The standards for kaempferol, quercetin, isorhamnetin, apigenin, luteolin and fisetin were obtained from Sigma (St Louis, MO, USA). Celery was purchased in a local supermarket.

\section{Subjects and dietary assessment}

Ninety-two students from the university campus were recruited. All subjects completed the study. The mean age of the subjects was $24 \cdot 16$ years with a range of 20-28 years, and the mean BMI was $21.31 \mathrm{~kg} / \mathrm{m}^{2}$ with a range of $16.02-32.46 \mathrm{~kg} / \mathrm{m}^{2}$. Inclusion criteria were as follows: being healthy (not using medicines, such as aspirin, antihypertension drugs and especially antibiotics); not suffering from metabolic diseases; not being pregnant or breastfeeding; not following a special diet. All subjects gave written informed consent after having been informed about the study aims and procedures. The study was conducted according to the guidelines laid down in the Declaration of Helsinki, and the study protocol was approved by the ethical committee of Harbin Medical University.

Data on the flavonoid (quercetin, isorhamnetin, kaempferol, apigenin and luteolin) content in food were taken from our earlier study, the results of which are described in detail elsewhere (manuscript submitted). We used the method of Cao et al. ${ }^{(15)}$ to determine the content of selected flavonoids (quercetin, isorhamnetin, kaempferol, apigenin and luteolin) in 100 edible vegetables and fruits in Harbin, China. Thus, we have established a flavonoid (quercetin, isorhamnetin, kaempferol, apigenin and luteolin) database for Harbin, China. Regarding food outside our database, we referred to the data in the United States Department of Agriculture database. For the present evaluation, the food content of quercetin, isorhamnetin, kaempferol, apigenin and luteolin was investigated.

Nutritional data for the participants were collected by means of 7-d dietary records (7-d DR) using a picture booklet for the estimation of portion sizes. The participants were given instructions by an experienced nutritionist at the start of the protocol period. During the protocol phase, all subjects were recontacted at least once by telephone. The flavonoid intake was obtained by multiplying the food content of flavonoids by the daily consumption of food items. Individual flavonoid intake data were calculated as the mean over the 7-d period (7-d DR). Data were examined for possible recording errors by checking for very high and very low intakes.

\section{Collection and preparation of blood samples}

Fasting blood samples were obtained from each participant at the end of the protocol period. Venous blood samples were drawn into tubes containing EDTA and centrifuged (4000 rpm, $15 \mathrm{~min}$ ). Plasma samples were obtained and stored at $-80^{\circ} \mathrm{C}$ until analysis.

A previously described methodology ${ }^{(16)}$ was applied to the hydrolysis and extraction procedure of the selected flavonoid compounds. To a volume of $1 \mathrm{ml}$ plasma, $110 \mu \mathrm{l}$ sodium acetate $(0.78 \mathrm{~mol} / \mathrm{l})$ and $100 \mu \mathrm{l}$ ascorbic acid $(0.1 \mathrm{~mol} / \mathrm{l})$ were added. About $100 \mu \mathrm{l}$ fisetin $(2 \mu \mathrm{g} / \mathrm{ml})$ was added as an internal standard. After adding $232.5 \mathrm{U}$ sulphatase and vortex mixing for $1 \mathrm{~min}$, the mixture was incubated for $30 \mathrm{~min}$ at $37^{\circ} \mathrm{C}$. The incubation was terminated by adding $20 \mu \mathrm{l}$ phosphoric acid $(85 \%)$. The flavonoids were extracted using a Bond Elut C18 solid-phase extraction column (Oasis HLB, Waters Corp., Milford, MA, USA). The flavonoids of matrix interference were eluted with $1 \mathrm{ml}$ methanol-2\% acetic acid aqueous solution (v/v, 5/95). The flavonoid compounds were eluted with $2 \mathrm{ml}$ methanol and dried under vacuum. The residue was then resolved in $100 \mu \mathrm{l}$ methanol/0.1\% phosphoric acid aqueous solution $(3 / 2, \mathrm{v} / \mathrm{v})$.

\section{HPLC analysis}

The plasma concentrations of quercetin, kaempferol, isorhamnetin, apigenin and luteolin were measured using a HPLC (Waters 600) ${ }^{(16)}$. The HPLC system included a Waters 2996 diode array detector and a Waters 2465 electrochemical detector. For HPLC analysis, $30 \mu l$ of the final solution were injected onto a Hypersil C18 ODS column ( $250 \mathrm{~mm} \times 4.6 \mathrm{~mm}$, particle size $5 \mu \mathrm{m}$, Thermo Corporation) maintained at $40^{\circ} \mathrm{C}$. The mobile phase consisted of two solvents (A/B, v/v, 3/2): A (distilled water adjusted to $\mathrm{pH}$ 2.25 with phosphoric acid) and $\mathrm{B}$ (methanol). The flow rate was $1 \mathrm{ml} / \mathrm{min}$. We used isocratic elution. Chromatograms were monitored by the electrochemical detector at a voltage of $1100 \mathrm{mV}$. Direct-current voltage amperometric mode was used. For onward determination, UV spectra were produced using the diode array detector. The acquisition and processing of chromatography data were achieved using Waters' Empower software, and the substances were identified by comparison with the retention time of the flavonoid standards and by analysis of the spectra.

Mean recovery of flavonoid standards added to plasma ( $n$ 3) amounted to $104.25 \%$ (quercetin), $97.31 \%$ (kaempferol), $95.93 \%$ (isorhamnetin), $97.26 \%$ (luteolin) and $93.49 \%$ (apigenin). The method showed good reproducibility with coefficients of variation of $6.84 \%$ (quercetin), $6.01 \%$ (kaempferol), $5.92 \%$ (isorhamnetin), $5.77 \%$ (luteolin) and $7.98 \%$ (apigenin). 


\section{The dietary intervention study}

Supplementary to the 7-d dietary investigation, we randomly chose twenty students (ten males and ten females) from the above subjects as the subsample for the dietary intervention study. These subjects were in the age range of 24-27 years, and the mean BMI was $20.81 \mathrm{~kg} / \mathrm{m}^{2}$. The subjects were requested to adhere to an apigenin- and luteolin-free diet (because luteolin is the main metabolite of apigenin ${ }^{(17)}$ ) for $3 \mathrm{~d}$ before the experimental day. Early in the morning on the experimental day, a fasting blood sample was collected and the subjects then consumed celery leaf at a dose of $2 \mathrm{~g} / \mathrm{kg}$ bw. Afterwards, blood samples were collected at 4, 6, $7,8,9,10,11$ and $28 \mathrm{~h}$ after celery leaf consumption, of these blood samples, the one collected at $28 \mathrm{~h}$ also served as the fasting blood sample (on the day after the experimental day). And at the same time, we gave the subjects bread for three meals on the experimental day in order to meet their basic metabolic needs. The blood samples were prepared and analysed using the same method as described above.

\section{Statistical analysis}

All analyses were performed with the statistics software SPSS version 13.0 (SPSS, Inc., Chicago, IL, USA). Statistical significance was set at $P<0 \cdot 05$. All of the statistical tests were two tailed. The results are presented as means, standard deviation (SD), minimum (Min) and maximum (Max) and percentiles $(10 \%$ and $90 \%)$. Deviation of the flavonoid intake estimates and plasma concentrations from normal distribution were tested by the Kolmogorov-Smirnov test. Non-parametric tests were used in the statistical analyses of the data. Spearman correlation was performed to analyse the associations of the dietary intakes with plasma concentrations. Regression analysis was applied for normally distributed data.

\section{Results}

The dietary flavonoid intakes (7-d DR) are shown in Table 1. The mean intake of the flavonols quercetin, kaempferol and isorhamnetin was $13.58,14.97$ and $12.31 \mathrm{mg} / \mathrm{d}$, respectively. The average intake of the flavones apigenin and luteolin was 4.23 and $8.08 \mathrm{mg} / \mathrm{d}$, respectively. A large range was found for the estimated individual mean flavonoid intake. The mean intake of quercetin, kaempferol, isorhamnetin, apigenin and luteolin ranged from 0-50.74, 0-70.48, 0-40.76, $0-17.95$ and $0-33.13 \mathrm{mg} / \mathrm{d}$, respectively. The single flavonoid intake in some subjects was zero. For all five flavonoids, the 10th percentile was above zero. For all subjects, the main sources of flavonols intake were apple $(13 \%)$, potato $(8 \%)$ and celery $(7 \%)$. The dietary flavones were mainly provided by celery $(9 \%)$ and eggplant $(6 \%)$.

The plasma flavonoids were determined after enzymatic hydrolysis, and only aglycones were detectable in each of the plasma samples. The mean plasma concentrations of quercetin, kaempferol, isorhamnetin, apigenin and luteolin are shown in Table 1 . The average values of quercetin, kaempferol, isorhamnetin, apigenin and luteolin were 80.23, 57.86, $39.94,10.62$ and $99.90 \mathrm{nmol} / \mathrm{l}$, respectively. It should be noted that the plasma flavonoid concentrations below the limit of detection have been denoted as zero when calculating their mean values. On average, plasma concentrations below the limit of detection were measured for quercetin in eighteen participants $(20 \%)$, for kaempferol in thirty-six participants $(39 \%)$, for isorhamnetin in twenty participants $(22 \%)$, for apigenin in twenty-five participants $(27 \%)$ and for luteolin in thirty-two participants $(35 \%)$. In contrast, the maximum values suggested that comparably high plasma concentrations could be found for all of the five flavonoids.

For all five plasma flavonoids, spearman correlation analyses revealed statistically significant relationships between

Table 1. The mean 7-d dietary intake $(\mathrm{mg} / \mathrm{d})$ derived from 7-d dietary records and fasting plasma concentrations (nmol/l) of quercetin $(\mathrm{Qu})$, kaempferol $(\mathrm{Ka})$, isorhamnetin (Is), apigenin (Ap) and luteolin (Lu) in ninety-two students

(Mean, median, standard deviations, minimum and maximum values)

\begin{tabular}{|c|c|c|c|c|c|c|c|}
\hline & \multirow[b]{2}{*}{ Mean } & \multirow[b]{2}{*}{ SD } & \multirow[b]{2}{*}{ Median } & \multirow[b]{2}{*}{ Minimum } & \multicolumn{2}{|c|}{ Percentiles (\%) } & \multirow[b]{2}{*}{ Maximum } \\
\hline & & & & & 10 & 90 & \\
\hline \multicolumn{8}{|c|}{ The mean 7-d dietary intake (mg/d) } \\
\hline Qu & $13 \cdot 58$ & $11 \cdot 18$ & $10 \cdot 76$ & 0 & 1.02 & $30 \cdot 20$ & $50 \cdot 74$ \\
\hline $\mathrm{Ka}$ & 14.97 & $13 \cdot 72$ & $10 \cdot 94$ & 0 & $2 \cdot 10$ & 31.08 & $70 \cdot 48$ \\
\hline Is & $12 \cdot 31$ & $9 \cdot 59$ & $10 \cdot 84$ & 0 & $2 \cdot 45$ & $23 \cdot 18$ & $40 \cdot 76$ \\
\hline$\Sigma$ Fls & $40 \cdot 87$ & $28 \cdot 80$ & $38 \cdot 66$ & 0 & $7 \cdot 12$ & $80 \cdot 36$ & $134 \cdot 35$ \\
\hline Ap & $4 \cdot 23$ & $3 \cdot 23$ & 3.54 & 0 & 1.05 & $7 \cdot 97$ & $17 \cdot 95$ \\
\hline Lu & 8.08 & $5 \cdot 64$ & $7 \cdot 41$ & 0 & $2 \cdot 17$ & $15 \cdot 07$ & $33 \cdot 13$ \\
\hline$\Sigma$ Fes & $12 \cdot 31$ & 8.48 & 11.03 & 0 & 3.79 & 22.54 & $46 \cdot 51$ \\
\hline$\Sigma F d s$ & $53 \cdot 19$ & $36 \cdot 09$ & $49 \cdot 47$ & 0 & $11 \cdot 22$ & $98 \cdot 60$ & $176 \cdot 75$ \\
\hline \multicolumn{8}{|c|}{ Fasting plasma concentrations (nmol/l) } \\
\hline Qu & $80 \cdot 23$ & $81 \cdot 73$ & 53.95 & $-{ }^{*}$ & $-{ }^{*}$ & $222 \cdot 72$ & $254 \cdot 65$ \\
\hline $\mathrm{Ka}$ & $57 \cdot 86$ & $60 \cdot 88$ & $38 \cdot 00$ & $-{ }^{*}$ & $-{ }^{*}$ & $154 \cdot 61$ & $173 \cdot 26$ \\
\hline Is & 39.94 & $45 \cdot 01$ & 20.09 & $-{ }^{*}$ & $-{ }^{*}$ & 107.96 & $156 \cdot 87$ \\
\hline$\Sigma$ Fls & 178.03 & $130 \cdot 37$ & 159.92 & $-{ }^{*}$ & $9 \cdot 17$ & 363.82 & 468.92 \\
\hline$A p$ & $10 \cdot 62$ & $12 \cdot 28$ & $6 \cdot 07$ & $-{ }^{*}$ & $-^{*}$ & 30.98 & $60 \cdot 32$ \\
\hline Lu & 99.90 & $97 \cdot 65$ & $77 \cdot 46$ & $-{ }^{*}$ & $-^{*}$ & 221.55 & 287.55 \\
\hline$\Sigma F e s$ & $108 \cdot 08$ & $102 \cdot 38$ & $80 \cdot 14$ & $-{ }^{*}$ & $-^{*}$ & $248 \cdot 10$ & $322 \cdot 76$ \\
\hline$\Sigma F d s$ & $286 \cdot 11$ & 194.69 & $289 \cdot 21$ & $-^{*}$ & $13 \cdot 87$ & 555.41 & $750 \cdot 84$ \\
\hline
\end{tabular}

Fls, flavonols; Fes, flavones; Fds, flavonoids.

*Below the limit of detection (results below the limit of detection have been denoted by the value zero). 
single and total flavonoid intakes and their corresponding fasting plasma concentrations (Table 2). For the results of the 7-d DR, correlation coefficients ranged from 0.33 (isorhamnetin) to 0.51 (quercetin; $P<0.05$ ). Correlation coefficients for quercetin intake $v$. the sum of plasma flavonol concentrations, isorhamnetin intake $v$. the sum of plasma flavonol concentrations, apigenin intake $v$. the sum of plasma flavonoid concentrations and luteolin intake $v$. the sum of plasma flavonoid concentrations were $0.52(P=0.000), 0.39$ $(P=0.000), 0.55(P=0.000)$ and $0.56(P=0.000)$, respectively. We found a weak correlation between the dietary intake of isorhamnetin and the fasting plasma flavonol concentrations.

With respect to the sum of flavonols and flavones, spearman correlation coefficients were within the range given for the individual flavonoid compounds and for the sum of flavonoids, excluding the range given for the single compounds. For the sum of flavonols, the relationship between the dietary intake and the plasma concentration is shown in Fig. 1, including the results of the regression analysis. The dietary intake of total flavonols showed a significantly positive correlation with the corresponding plasma concentration ( $r$ 0.48, $P=0.000$ ), which indicated that individual variation in dietary flavonol intake contributed to the variation in the plasma flavonol concentrations. Similarly, for the sum of flavonoids, the association of the dietary intake with the plasma concentration is shown in Fig. 1. The dietary intake of total flavonoids was also positively associated with the corresponding plasma concentration $(r 0.58, P=0 \cdot 000)$.

In the intervention study, before the consumption of celery leaf, apigenin was not detected in fasting plasma samples of subjects. After eating celery leaf, the plasma apigenin concentration showed an upward trend (Table 3) and reached a maximum concentration at $7.70 \pm 0.82 \mathrm{~h}$. The area under the blood concentration time curve from 0 to $28 \mathrm{~h}$ was 89.02 (SD 40.57) $\mathrm{min} \times \mu \mathrm{mol} / \mathrm{l}$. For all participants, the plasma apigenin concentration fell within $28 \mathrm{~h}$ to below the limit of detection $(2.32 \mathrm{nmol} / \mathrm{l})$. A large range of variation $(0-396.25 \mathrm{nmol} / \mathrm{l})$ was found among the subjects for plasma apigenin concentrations determined between 0 and $28 \mathrm{~h}$.

\section{Discussion}

The HPLC analysis in the present study allowed the simultaneous quantification of five different flavonoids in plasma samples originating from subjects adhering to their habitual diet. Plasma samples collected at the end of the protocol period reflected the 7-d food registration, which may result in a reduction in the number of samples required and increase the strength of each analysis as it reflects an average consumption of flavonoids over $7 \mathrm{~d}$ instead of in a single day only, thus reducing any reporting errors or incomplete plasma collection.

In the present study, the range of the mean dietary flavonoid intake of the subjects was wide. The mean flavonol intake $(28.55 \mathrm{mg} / \mathrm{d}$ for quercetin and kaempferol) was much higher than that reported previously $(12.5 \mathrm{mg} / \mathrm{d}$ for quercetin and kaempferol $^{(18)}, 22.6 \mathrm{mg} / \mathrm{d}$ for flavonols and flavones $\left.{ }^{(19)}\right)$. This suggests that the subjects consumed a high level of flavonoidrich foodstuff. The inter-individual variation in intake of flavonols and flavones was comparably high, which reflected the variation in the subjects' diet. The dietary flavonoid intake strongly depends on food choice habits and the consumption of food, and the latter is influenced by the method of analysis of the flavonoids and factors affecting flavonoid content in food such as light, cultivar, growing location, environmental conditions, degree of ripeness and so on. Although data, such as the United States Department of Agriculture database for flavonoids, enable and facilitate the assessment of daily flavonoid intake $^{(20-22)}$, different populations in different countries consume different types of plant foods. Therefore, our previous laboratory detection of flavonoids using the method of Cao et al. ${ }^{(15)}$ provides the basis for the evaluation of the dietary flavonoid intake presented here. And it can be assumed that the calculated values are fairly good assessment of the true intake and comparable to other recent studies.

After polyphenol consumption, plasma concentrations varied widely according to the nature of the polyphenol and the food source. They were of the order of $0.3-0.75 \mu \mathrm{mol} / 1$ after consumption of $80-100 \mathrm{mg}$ quercetin equivalent administered in the form of apples, onions or meals rich in plant products $^{(23-25)}$. Isoflavones are certainly the best absorbed flavonoids: plasma concentrations of $1.4-4 \mu \mathrm{mol} / 1$ were obtained between 6 and $8 \mathrm{~h}$ in adults who consumed relatively low quantities of soya derivatives supplying $50 \mathrm{mg}$ isoflavones $^{(26-28)}$. The situation for the diversity of flavonoids is similar at the absorption site, although the information has increased considerably in recent years ${ }^{(29)}$. It is evident that the bioavailability of quercetin, and most likely of other

Table 2. Correlation between the fasting plasma flavonoid concentrations $(\mathrm{nmol} / \mathrm{l})$ and the mean $7-\mathrm{d}$ dietary flavonoid intake $(\mathrm{mg} / \mathrm{d})$ derived from 7 -d dietary records obtained in ninety-two students

(Spearman correlation coefficients)

\begin{tabular}{|c|c|c|c|c|c|c|c|c|}
\hline \multirow{2}{*}{ Fds intake } & \multicolumn{8}{|c|}{ Fasting plasma concentrations } \\
\hline & $\mathrm{Qu}$ & $\mathrm{Ka}$ & Is & $\Sigma F l s$ & Ap & $\mathrm{Lu}$ & $\Sigma$ Fes & $\Sigma F d s$ \\
\hline Qu & $0.51^{*}$ & 0.19 & 0.31 & $0.52^{*}$ & 0.57 & 0.56 & 0.57 & 0.65 \\
\hline $\mathrm{Ka}$ & 0.11 & $0.44^{*}$ & 0.33 & 0.39 & 0.28 & 0.29 & 0.32 & 0.43 \\
\hline Is & 0.09 & 0.45 & $0.33^{*}$ & $0.39^{*}$ & 0.28 & 0.30 & 0.32 & 0.41 \\
\hline$\Sigma$ Fls & 0.27 & 0.40 & 0.35 & $0.48^{*}$ & 0.41 & 0.46 & 0.48 & 0.57 \\
\hline$A p$ & 0.32 & 0.41 & 0.38 & 0.49 & $0.42^{*}$ & 0.40 & 0.41 & $0.55^{\star}$ \\
\hline $\mathrm{Lu}$ & 0.23 & 0.47 & 0.39 & 0.48 & 0.37 & $0.44^{*}$ & 0.46 & $0.56^{*}$ \\
\hline$\Sigma$ Fes & 0.27 & 0.46 & 0.39 & 0.50 & 0.41 & 0.44 & $0.46^{*}$ & 0.57 \\
\hline$\Sigma F d s$ & 0.27 & 0.43 & 0.37 & 0.50 & 0.40 & 0.46 & 0.48 & $0.58^{*}$ \\
\hline
\end{tabular}

Qu, quercetin; Ka, kaempferol; Is, isorhamnetin; Ap, apigenin; Lu, luteolin; Fls, flavonols; Fes, flavones; Fds, flavonoids. ${ }^{\star} P<0.05$ (two tailed) 

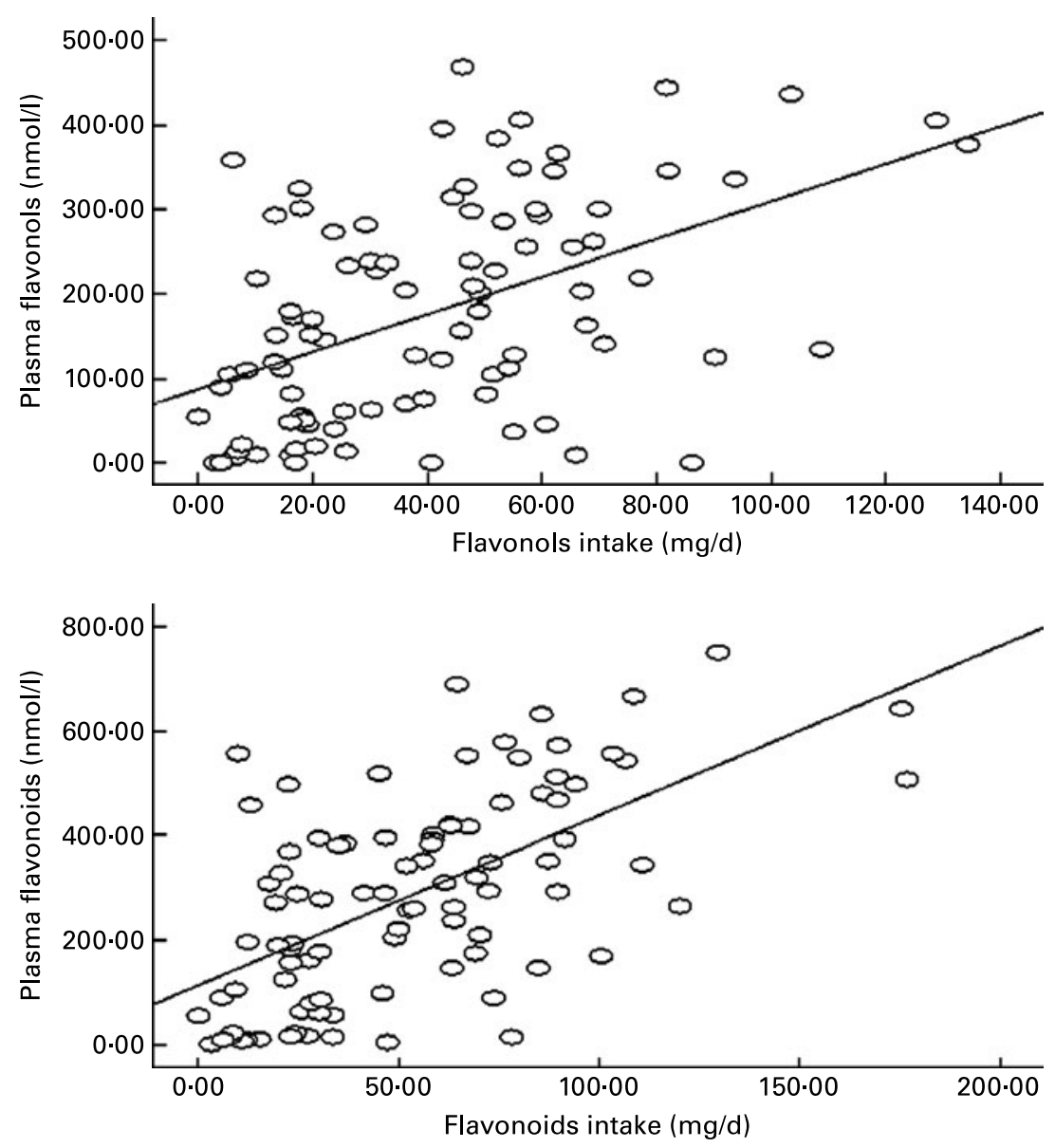

Fig. 1. Relationship between the dietary intake and the corresponding plasma concentration in ninety-two students, for the sum of flavonols (quercetin, kaempferol and isorhamnetin) $\left(r^{2} 0.24, P=0.000\right)$ and for the sum of flavonoids (quercetin, kaempferol, isorhamnetin, apigenin and luteolin) $\left(r^{2} 0.36, P=0.000\right.$; all data here fit a normal distribution).

flavonoids as well, is greatly affected by the type and binding site of the sugar moieties ${ }^{(30)}$. Flavonoid absorption rates are subject to considerable inter-individual variability ${ }^{(31)}$, which is illustrated in the present study by the large variation observed in plasma flavonoid concentrations.

A wide range of variation has been shown in the reported data on flavonoid concentrations in individual fasting plasma samples. To our knowledge, data on plasma quercetin

Table 3. Plasma apigenin concentration (nmol/l) in twenty students by time (h) after eating celery leaf

(Mean, median, standard deviations, minimum and maximum values)

\begin{tabular}{|c|c|c|c|c|c|}
\hline $\begin{array}{l}\text { Time } \\
\text { (h) }\end{array}$ & $\begin{array}{l}\text { Mean } \\
(\mathrm{nmol} / \mathrm{l})\end{array}$ & $\begin{array}{c}\mathrm{SD} \\
(\mathrm{nmol} / \mathrm{l})\end{array}$ & $\begin{array}{l}\text { Median } \\
(\mathrm{nmol} / \mathrm{l})\end{array}$ & $\underset{(\mathrm{nmol} / \mathrm{l})}{\text { Minimum }}$ & $\begin{array}{c}\text { Maximum } \\
(\mathrm{nmol} / \mathrm{l})\end{array}$ \\
\hline 0 & - * & $-*$ & $-*$ & $-*$ & $-*$ \\
\hline 4 & $58 \cdot 12$ & 30.53 & 73.45 & $6 \cdot 88$ & $101 \cdot 76$ \\
\hline 6 & $134 \cdot 20$ & $64 \cdot 71$ & 111.57 & $58 \cdot 76$ & 292.57 \\
\hline 7 & $190 \cdot 41$ & 98.54 & $147 \cdot 83$ & $103 \cdot 61$ & 361.53 \\
\hline 8 & 126.99 & 105.66 & $110 \cdot 67$ & 23.85 & $396 \cdot 25$ \\
\hline 9 & 125.95 & 97.40 & $120 \cdot 77$ & $20 \cdot 67$ & $350 \cdot 27$ \\
\hline 10 & 90.96 & $75 \cdot 16$ & $105 \cdot 54$ & 23.85 & $280 \cdot 27$ \\
\hline 11 & 62.50 & 39.33 & $57 \cdot 18$ & $19 \cdot 60$ & $100 \cdot 25$ \\
\hline 28 & $-^{*}$ & $-^{*}$ & $-^{*}$ & $-^{*}$ & $-^{*}$ \\
\hline
\end{tabular}

*Below the limit of detection. concentration are mainly derived from studies in which rather large amounts of foods rich in quercetin were consumed. In the present study, fasting plasma concentrations of quercetin were found to be between 0 and $254.65 \mathrm{nmol} / 1$ as compared with the values of $0-142 \mathrm{nmol} / \mathrm{l}$ reported previously ${ }^{25,32)}$. The mean fasting plasma concentration of quercetin $(80.23 \mathrm{nmol} / \mathrm{l})$ was higher than that for kaempferol, isorhamnetin and apigenin, but lower than the value of luteolin, most likely as a consequence of the lower dietary quercetin intake. Although quercetin is widely distributed in plant species, it is generally present only in low concentrations, except for specific plant foods with very high quercetin content such as onions, cruciferous vegetables and some berries $^{(33)}$. Endogenous metabolism resulting in configurational changes in the flavonoid aglycones has been reported, and the major proportion of the excreted flavonoids probably results from flavonoids absorbed from the diet. Isorhamnetin, however, may also result from endogenous metabolism of quercetin by catechol- $O$-methyltransferase ${ }^{(34)}$.

A potential limitation of the present study was that the samples of about one-fifth to one-third of the subjects contained non-detectable concentrations of individual flavonoids. However, this did not limit our ability to detect significant correlations between the intake and plasma concentrations. In the present study, statistically significant correlations 
between the mean intake (7-d DR) and the fasting plasma concentrations of quercetin ( $r$ 0.51), kaempferol $(r 0.44)$ and the sum of flavonoids $(r 0.58)$ were observed for the normal dietary intake. It has already reported in supplementation studies that the difference in the intake of quercetin and kaempferol can be distinguished by their concentrations in plasma and urine ${ }^{(35)}$. Linseisen et al. ${ }^{(19)}$ analysed the relationship between the mean flavonoid intake (7-d DR) and fasting plasma concentrations, and found statistically significant correlations between the mean intake and fasting plasma concentrations of quercetin $(r 0.30)$ and kaempferol $(r$ 0.46). Noroozi et al. ${ }^{(36)}$ quantified the relationship between the dietary quercetin intake and the plasma and urine concentrations in a study supplementing the usual diet with meals containing high levels of flavonols, and found statistically significant correlations between dietary quercetin intake and the quercetin concentration in plasma $(r 0.74)$ and urine $(r 0.81)$. Nielsen et al. ${ }^{(37)}$ have reported significant correlations between the mean intake of fruits, berries and vegetables and the urinary concentration of flavonoids $(r$ 0.308), and between the excretion of kaempferol and intake of tea $(r 0.485)$. The difference in the degree of significance probably results from considerable individual variation in response to intake and other dietary factors influencing digestion and absorption.

In the intervention study, it was found that the plasma apigenin concentration of all subjects decreased to below the detection limit at $28 \mathrm{~h}$ after eating celery leaf. Meyer et al. ${ }^{(38)}$ have observed that after the ingestion of a bolus, the plasma apigenin concentration rose and fell within $28 \mathrm{~h}$ to below the limit of detection $(2 \cdot 3 \mathrm{nmol} / \mathrm{l})$. Manach et al. ${ }^{(25)}$ reported that quercetin concentrations fell to basal levels at $20 \mathrm{~h}$ in the volunteers after the consumption of a complex meal rich in plant products. The present result demonstrates that the plasma concentrations of apigenin and quercetin only reflect short-term intake rather than the lifelong diet. Further investigation is required to determine whether after the ingestion of kaempferol, isorhamnetin and luteolin, their plasma concentrations fall to below the limit of detection within $28 \mathrm{~h}$. Moreover, considering the dietary intake on the day before the collection of blood samples, the fasting plasma flavonoid concentrations were better markers of short-term intake.

The present study suggests that five different dietary flavonoids were measurable in plasma from subjects consuming their habitual diet, and that the sum of flavonoids extracted in plasma is associated with the dietary intake. Plasma flavonoids may therefore be a valid biomarker of flavonoid intake. This is supported by the positive correlation between the dietary flavonoid intake and the corresponding plasma concentration. Even though the present data suggest that the plasma total flavonols and flavonoids may be the best markers for the corresponding intake, individual flavonoids may serve as indicators of specific flavonoid intake. The strength of the correlation between the dietary flavonoid intake and the corresponding plasma concentration seems comparable to other nutritional biomarkers. Plasma carotenoids have often shown only a weak correlation with the intake of fruits and vegetables $^{(39)}$. The impact of other parameters (besides diet) affecting plasma concentrations, such as the bioavailability and the metabolic handling of the compounds, needs to be defined more precisely.
In conclusion, fasting plasma flavonoid concentrations are significantly correlated with their corresponding dietary intake, and fasting plasma flavonoid concentrations seem to be potential biomarkers of their dietary intake. Single fasting plasma flavonoid values truly reflect the short-time dietary intake. However, more information on the dose dependency of flavonoid excretion is needed before the validity of plasma flavonoids as biomarkers of intake can be established. The validity of plasma flavonoids as biomarkers of the ordinary dietary intake should be further investigated in intervention and cohort studies.

\section{Acknowledgements}

Financial support from the Education Department of Heilongjiang Province (11541157) is gratefully acknowledged. We would like to thank all the subjects for their participation. All authors have contributed to the study and accept responsibility for the content of the paper. There are no conflicts of interest. X. J. Z. designed and supervised the study. J. C. analysed the study data and contributed to writing the manuscript. J. C., W. C. and Y. Z. supervised the community survey and the plasma flavonoid assays.

\section{References}

1. Lwashina $\mathrm{T}$ (2000) The structure and distribution of the flavonoids in plants. J plant res 113, 287-299.

2. Middleton EJ, Kandaswami C \& Theoharides TC (2000) The effects of plant flavonoids on mammalian cells: implications for inflammation, heart disease, and cancer. Pharmacol Rev 52, 673-751.

3. Arts ICW \& Hollman PCH (2005) Polyphenols and disease risk in epidemiological studies. Am J Clin Nutr 81, Suppl. 1, 317S-325S.

4. Geleijnse JM, Launer LJ, Van der Kuip DA, et al. (2002) Inverse association of tea and flavonoid intakes with incident myocardial infarction: The Rotterdam Study. Am J Clin Nutr 75, 880-886.

5. Erdman JW Jr, Carson L, Kwik-Uribe C, et al. (2008) Effects of cocoa flavanols on risk factors for cardiovascular disease. Asia Pac J Clin Nutr 17, Suppl. 1, 284-287.

6. Knekt P, Isotupa S, Rissanen H, et al. (2000) Quercetin intake and the incidence of cerebrovascular disease. Eur J Clin Nutr 54, 415-417.

7. Sesso HD, Gaziano JM, Liu S, et al. (2003) Flavonoid intake and the risk of cardiovascular disease in women. Am J Clin Nutr 77, 1400-1408.

8. He S, Sun C \& Pan Y (2008) Red wine polyphenols for cancer prevention. Int J Mol Sci 9, 842-853.

9. Goldbohm RA, Hertog MGL, Brants HAM, et al. (1998) Intake of flavonoids and cancer risk: a prospective cohort study. In Polyphenols in Food, pp. 159-166 [R Armado, H Andersson, $\mathrm{S}$ Bardócz and F Serra, editors]. Luxembourg: Office for Official Publications of the European Communities.

10. Beecher GR (2003) Overview of dietary flavonoids: nomenclature, occurrence and intake. J Nutr 133, 3048S-3054S.

11. Crews H, Alink G, Andersen R, et al. (2001) A critical assessment of some biomarker approaches linked with dietary intake. Br J Nutr 86, Suppl. 1, S5-S35.

12. Erlund I, Meririnne E, Alfthan G, et al. (2001) Plasma kinetics and urinary excretion of the flavanones naringenin and hesperetin in humans after ingestion of orange juice and grapefruit juice. J Nutr 131, 235-241. 
13. Ito H, Gonthier MP, Manach C, et al. (2005) Polyphenol levels in human urine after intake of six different polyphenol-rich beverages. Br J Nutr 94, 500-509.

14. Erlund I, Kosonen T, Alfthan G, et al. (2000) Pharmacokinetics of quercetin from quercetin aglycone and rutin in healthy volunteers. Eur J Clin Pharmacol 56, 545-553.

15. Cao J, Zhao XJ, Wu K, et al. (2008) Simultaneous determination of five flavonoid compounds in vegetables and fruits by high performance liquid chromatography. Chinese J Prev Med Inf 7, 525-527.

16. Zhao XJ, Wu K, Zhao Y, et al. (2007) High performance liquid chromatographic method for the determination of apigenin and selected flavonoids in human plasma using electrochemical detection. Chinese J Anal Chem 10, 1517-1520.

17. Nielsen SE, Breinholt V, Justesen U, et al. (1998) In vitro biotrabsformation of flavonoids by rat liver microsomes. Xenobiotica 28, 389-401.

18. Linseisen J, Radtke J \& Wolfram G (1997) Flavonoid intake of adults in a Bavarian subgroup of the national food consumption survey. Z Ernahrungswiss 36, 403-412.

19. Radtke J, Linseisen J \& Wolfram G (2002) Fasting plasma concentrations of selected flavonoids as markers of their ordinary dietary intake. Eur J Nutr 41, 203-209.

20. Scalbert A \& Williamson G (2000) Dietary intake and bioavailability of polyphenols. J Nutr 130, Suppl. 8S, 2073S-2085S.

21. Kinoshita T, Lepp Z \& Chuman H (2005) Construction of a novel database for flavonoids. J Med Invest 52, 291-292.

22. Peterson J \& Dwyer J (2000) An informatics approach to flavonoid database development. J Food Compos Anal 13, 441-454.

23. Graefe EU, Wittig J, Mueller S, et al. (2001) Pharmacokinetics and bioavailability of quercetin glycosides in humans. J Clin Pharmacol 41, 492-499.

24. Hollman PC, van Trijp JM, Buysman MN, et al. (1997) Relative bioavailability of the antioxidant flavonoid quercetin from various foods in man. FEBS Lett 418, 152-156.

25. Manach C, Morand C, Crespy V, et al. (1998) Quercetin is recovered in human plasma as conjugated derivatives which retain antioxidant properties. FEBS Lett 426, 331-336.

26. Xu X, Wang HJ, Murphy PA, et al. (1994) Daidzein is a more bioavailable soymilk isoflavone than is genistein in adult women. J Nutr 124, 825-832.

27. King RA \& Bursill DB (1998) Plasma and urinary kinetics of the isoflavones daidzein and genistein after a single soy meal in humans. Am J Clin Nutr 67, 867-872.
28. Shelnutt SR, Cimino CO, Wiggins PA, et al. (2002) Pharmacokinetics of the glucuronide and sulfate conjugates of genistein and daidzein in men and women after consumption of a soy beverage. Am J Clin Nutr 76, 588-594.

29. Ader P, Block M, Pietzsch S, et al. (2001) Interaction of quercetin glucosides with the intestinal sodium/glucose co-transporter (SGLT-1). Cancer Lett 162, 175-180.

30. Hollman PC, Bijsman MN, van Gameren Y, et al. (1999) The sugar moiety is a major determinant of the absorption of dietary flavonoid glycosides in man. Free Radic Res 31, 569-573.

31. Erlund I, Meririnne E, Alfthan G, et al. (2001) Plasma kinetics and urinary excretion of the flavanones naringenin and hesperetin in humans after ingestion of orange juice and grapefruit juice. J Nutr 131, 235-241.

32. Manach C, Morand C, Crespy V, et al. (1998) Quercetin is recovered in human plasma as conjugated derivatives which retain antioxidant properties. FEBS Lett 426, 331-336.

33. Kumpulainen JT, Lehtonen M \& Mattila P (1999) Trolox equivalent antioxidant capacity of average flavonoids intake in Finland. In Natural Antioxidants and Anticarcinogens in Nutrition, Proceedings of the Second International Conference on Natural Antioxidants, pp. 141-150 [JT Kumpulainen and JT Salonen, editors]. Cambridge: The Royal Society of Chemistry.

34. Zhu BT, Ezell EL \& Liehr JG (1994) Catechol-O-methyltransferasecatalyzed rapid $O$-methylation of mutagenic flavonoids. J Biol Chem 269, 292-299.

35. Nielsen SE, Kall M, Justesen U, et al. (1997) Human absorption and excretion of flavonoids after broccoli consumption. Cancer Lett 114, 173-174.

36. Noroozi M, Burns J, Crozier A, et al. (2000) Prediction of dietary flavonol consumption from fasting plasma concentration or urinary excretion. Eur J Clin Nutr 54, 143-149.

37. Nielsen SE, Freese R, Kleemola P, et al. (2002) Flavonoids in human urine as biomarkers for intake of fruits and vegetables. Cancer Epidemiol Biomarkers Prev 11, 459-466.

38. Meyer H, Bolarinwa A, Wolfram G, et al. (2006) Bioavailability of apigenin from apiin-rich parsley in humans. Ann Nutr Metab 50, $167-172$.

39. Zino S, Skeaff M, Williams S, et al. (1997) Randomised controlled trial of effect of fruit and vegetable consumption on plasma concentrations of lipids and antioxidants. $\mathrm{Br}$ Med $\mathrm{J}$ 314, 1787-1791. 